\title{
Bibliography
}

1. S. A. Amitsur, The identities of PI-rings, Proc. Amer. Math. Soc. vol. 4 (1953) pp. 27-34.

2. - Algebras over infinite fields, Proc. Amer. Math. Soc. vol. 7 (1956) pp. $35-48$.

3. I. Kaplansky, Rings with a polynomial identity, Bull. Amer. Math. Soc. vol. 54 (1948) pp. 575-580.

4. W. Krull, Jacobsonsches Radical und Hilbertscher Nullstellensatz, International Congress of Mathematicians, Cambridge, 1950, Conference in Algebra, pp. 56-64.

Hebrew University, JeRUSAlem

\section{A NOTE ON THE STONE-WEIERSTRASS THEOREM FOR QUATERNIONS ${ }^{1}$}

\author{
JOHN C. HOLLADAY
}

A result of M. H. Stone [1, p. 466], which is nicely presented by N. Dunford [2, p. 23], is as follows: Let $A$ be a closed subalgebra of the $B$-algebra $C(X)$ of all continuous real-valued functions on the compact Hausdorff space $X$. Then $A=C(X)$ if and only if $A$ distinguishes between every pair of distinct points of $X$, i.e., for every pair $x_{1} \neq x_{2}$ of points in $X$, there is an $f$ in $X$ such that $f\left(x_{1}\right) \neq f\left(x_{2}\right)$.

If one substitutes the word complex for the word real in the above statement, it becomes false. A well known counter example is obtained by letting $X$ be the set of complex numbers $z$ such that $|z| \leqq 1$ and letting $A$ be the subalgebra of functions which are analytic in the interior of $X$.

The purpose of this note is to show that if the word quaternion is substituted for the word real in the above statement, it remains valid. To be specific, let $A$ be a set of continuous quaternion-valued functions which satisfy the following conditions:

1. $A$ is complete.

2. Given a quaternion $q$, the function $f(x) \equiv q$ is in $A$.

3. If $f$ and $g$ are in $A$, then $f g$ and $f+g$ are in $A$.

Received by the editors November 18, 1956.

1 A small part of the work done under an AEC Predoctoral Fellowship at Yale University, year 1952-1953, under the kind and patient guidance of Dr. Charles E.
Rickart. 
If $A$ contains all continuous quaternion-valued functions, it obviously distinguishes between points. Letting $A$ distinguish between points, consider two arbitrary distinct points $x_{1}$ and $x_{2}$. Choose an element of $A$ which takes a different value at $x_{2}$ than at $x_{1}$. Multiply this function by an appropriate quaternion to obtain a function $f$ such that real part $\left[f\left(x_{1}\right)\right] \neq$ real part $\left[f\left(x_{2}\right)\right]$. But the real part of $f$ is $[f-i f i-j f j-k f k] / 4$ which is an element of $A$. Therefore, $A$ contains real valued functions which distinguish between points.

Since $A$ is complete, and is closed under multiplication, addition and subtraction, it follows that the set of all real-valued functions in $A$ is also complete and closed under these arithmetic operations. The Stone-Weierstrass Theorem implies that $A$ contains all continuous real-valued functions on $X$. Therefore, $A$ contains all continuous quaternion-valued functions on $X$.

\section{BIBLIOGRAPHY}

1. M. H. Stone, Applications of the theory of Boolean rings to general topology, Trans. Amer. Math. Soc. vol. 41 (1937) pp. 375-481.

2. N. Dunford, Spectral theory in abstract spaces and Banach algebras, Symposium on Spectral Theory and Differential Equations, pp. 1-26.

Los Alamos Scientific Laboratory and

YALE UNIVERSITY 